\title{
Detection of the Discrete Convexity of Polyominoes
}

\author{
Isabelle Debled-Rennesson ${ }^{1}$, Jean-Luc Rémy ${ }^{2}$, and Jocelyne Rouyer-Degli ${ }^{3}$ \\ 1 LORIA - Laboratoire LOrrain de Recherche en Informatique et ses Applications \\ Institut Universitaire de Formation des Maîtres de Lorraine \\ Campus Scientifique, B.P. 239, F54506 Vandœuvre-lès-Nancy \\ \{debled, remy, rouyer\}@loria.fr \\ 2 LORIA \\ Centre National de la Recherche Scientifique \\ Campus Scientifique, B.P. 239, F54506 Vandœuvre-lès-Nancy \\ ${ }^{3}$ LORIA \\ Université Henri Poincaré, Nancy 1 \\ Campus Scientifique, B.P. 239, F54506 Vandœuvre-lès-Nancy
}

\begin{abstract}
The convexity of a discrete region is a property used in numerous domains of computational imagery. We study its detection in the particular case of polyominoes. We present a first method, directly relying on its definition. A second method, which is based on techniques for segmentation of curves in discrete lines, leads to a very simple algorithm whose correctness is proven. Correlatively, we obtain a characterisation of lower and upper convex hulls of a discrete line segment. Finally, we evoke some applications of these results to the problem of discrete tomography.
\end{abstract}

Keywords: Discrete Convexity, Segmentation, Discrete line, Polyominoes, Discrete tomography

\section{Introduction}

Discrete convexity intervenes in numerous domains with regard to geometry and particularly to image processing [4]. It is an important property of plane figures which permits, for instance, methods for geometrical shapes regularisation. The notion of discrete convexity is strongly linked with the paradigm of discrete lines. This is underlined in this article where a simple and efficient algorithm for detection of polyomino convexity is presented. Polyominoes are objects in which any couple of cells may be linked through a path containing only horizontal and vertical moves (4-connectivity). After having checked the hv-convexity (cells of each column and each row are consecutive) of a polyomino $\mathrm{P}$, convexity is checked on the curve points of the border characterising it.

The proposed method uses a variant of the linear algorithm for segmentation of curves in straight lines given in $[7,6]$. The eventual non-convexity of $\mathrm{P}$ is detected by the algorithm, during its scanning of curves of the border of $\mathrm{P}$; if the whole border is scanned, $\mathrm{P}$ is convex. The proof of this algorithm uses a result on convex hull of a discrete segment presented in this article.

G. Borgefors, I. Nyström, and G. Sanniti di Baja (Eds.): DGCI 2000, LNCS 1953, pp. 491-504, 2000. (C) Springer-Verlag Berlin Heidelberg 2000 
This technique and methods directly coming from definitions of discrete convexity have been used for the study of the reconstruction of 2-dimensional discrete sets from their horizontal and vertical projections. In particular, we studied the reconstruction of convex polyominoes. The approaches we use are presented at the end of this article. This work enters in the more general framework of discrete tomography whose applications are numerous, particularly in data compression, image processing, or still in medical imagery for medical diagnosis help in radiography.

In the second section, we introduce miscellaneous definitions of discrete convexity. Then a first simple method, deduced from a definition, is proposed. In the following section, fundamental elements of discrete geometry are given so that we obtain a very efficient algorithm for convexity detection on hv-convex polyominoes. Then, we propose a use of these methods in discrete tomography. At last, a conclusion and further research prospects are given.

\section{Discrete Convexity}

Convexity is well defined in the continuous case but in the discrete one, several definitions exist. The studied discrete figures are finite 8-connected subsets of discrete points in the plane and are named discrete regions.

In 1970, Slansky [18] defines a discrete region as convex if and only if there is a convex (Euclidean) region whose image (after digitizing) is this discrete region. This definition depends on the digitizing process used.

On the other hand, Minsky and Papert [14] gave the following definition of the convexity of a discrete region $\mathrm{R}$ : $\mathrm{R}$ is convex if and only if there is no triplet of colinear discrete points $\left(c_{1}, c_{2}, c_{3}\right)$ such as $c_{1}$ and $c_{3}$ belong to $\mathrm{R}$ and $c_{2}$ belongs to the complementary of $\mathrm{R}$.

Then, Kim and Rosenfeld $[12,13,11]$ have given several equivalent definitions. They have shown that a discrete region $\mathrm{R}$ is convex

- on the one hand, if and only if its convex (Euclidean) hull does not contain any discrete point of the complementary of $R$.

- on the other hand, if and only if it fulfils the area property i.e. if and only if, for all points $\mathrm{p} 1$ and $\mathrm{p} 2$ of $\mathrm{R}, P(\mathrm{R}, \mathrm{p} 1, \mathrm{p} 2)$ does not possess any point of the complementary of $\mathrm{R}$, where $P(\mathrm{R}, \mathrm{a}, \mathrm{b})$ represents the polygon whose edges are made by the segment $\mathrm{ab}$ and the edges of $\mathrm{R}$ (see Fig. 1).

These last two definitions shall be used in the study of the convexity of polyominoes. We refer to Kim and Rosenfeld $[12,13,11]$ for a comparison of their definitions with Minsky and Papert's one.

A hv-convex polyomino is a polyomino whose cells of each column are consecutive (v-convexity) as well as those of each row (h-convexity).

Some immediate properties may be deduced from this definition:

- a hv-convex polyomino is a discrete region "without hole",

- a convex polyomino is hv-convex.

The convexity study of a polyomino so starts by checking its hv-convexity, this 

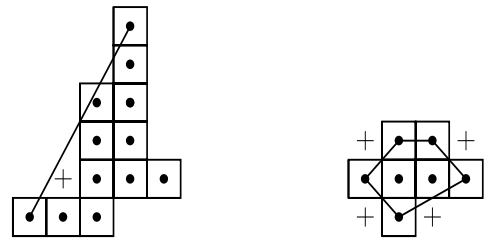

Fig. 1. On the left, a non-convex discrete region. On the right, a convex discrete region

is done through a simple scanning of each row [4]. In the following, we study the convexity of hv-convex polyominoes. It is clear that this study may therefore be reduced to the convexity study of the limit or border of the hv-convex polyominoes (see Sect. 3 and 4 ).

\section{Direct Use of the Area Property to Detect the Convexity of a $h v$-Convex Polyomino}

Let $\mathrm{T}$ be a hv-convex polyomino, anchored at $(k, l)$, i.e., containing in its first column a cell at row $k$ and in the last column a cell at row $l$. We call left limit of $\mathrm{T}$, and we note $\mathrm{L}$, the set of cells with minimal column index of each line. We define in the same way the right limit $\mathrm{R}$ of $\mathrm{T}$. We distinguish in $\mathrm{L}$ the higher part L1, included between the first line and the left anchorage $k$, and the lower part L2, included between the line $k$ and the last line. In the same way, we distinguish in $\mathrm{R}$ the parts $\mathrm{R} 1$ and $\mathrm{R} 2$ limited by the right anchorage $l$ (see Fig. 2).

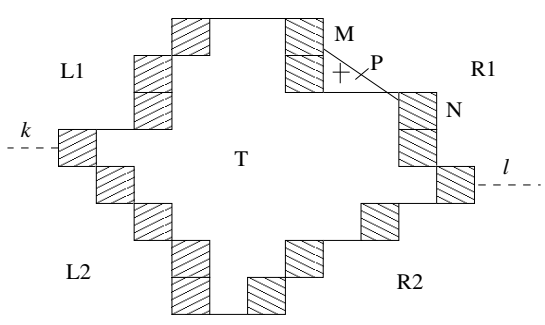

Fig. 2. Limits of the polyomino $\mathrm{T}$

To check that $\mathrm{T}$ is convex, we only have to apply the area property on L1, L2, R1, and R2. So T is convex if and only if, for every couple (M, N) of points of L1, L2, R1, or R2, and every $y$ included between the row indexes of $\mathrm{M}$ and $\mathrm{N}$, there is no discrete point whose row index $y$ is located between the segment MN (inclusive) and the polyomino $\mathrm{T}$ (exclusive). Let $\left(x_{1}, y_{1}\right)$ be the coordinates of $\mathrm{M},\left(x_{2}, y_{2}\right)$ the coordinates of $\mathrm{N}$. The coordinates of the point $\mathrm{P}$ of the segment 
MN with row index $y, y_{1}<y<y_{2}$, are $(x, y)$, with $x=x_{1}+\frac{\left(y-y_{1}\right)\left(x_{2}-x_{1}\right)}{y_{2}-y_{1}}$. In order that $\mathrm{T}$ be convex, it is necessary and sufficient that:

i. if $\mathrm{M}$ and $\mathrm{N}$ belong to L1 or to L2, the discrete point with coordinates ( $\lceil x\rceil, y)$, located to the right of the point $\mathrm{P}$, belongs to $\mathrm{T}$,

ii. if $\mathrm{M}$ and $\mathrm{N}$ belong to $\mathrm{R} 1$ or to $\mathrm{R} 2$, the discrete point with coordinates $(\lfloor x\rfloor, y)$, located to the left of the point $\mathrm{P}$, belongs to $\mathrm{T}$.

If the hv-convex polyomino $\mathrm{T}$ is represented by a 0-1 matrix whose dimension is $m * n$, the computation of its limits is in $\mathrm{O}(m+n), m$ and $n$ being the numbers of rows and columns of $\mathrm{T}$, respectively, and the tests on couples of points are in $\mathrm{O}\left(\min \left(m^{3}, n^{3}\right)\right)$. Indeed, when $m>n$, it is permitted to exchange the roles of columns and rows.

\section{Use of Discrete Lines to Detect the Convexity}

Let us consider a hv-convex polyomino included in a minimal rectangle Rec whose size is $m * n$. Let $\left(\left[\mathrm{A}, \mathrm{A}^{\prime}\right],\left[\mathrm{B}, \mathrm{B}^{\prime}\right],\left[\mathrm{C}, \mathrm{C}^{\prime}\right],\left[\mathrm{D}, \mathrm{D}^{\prime}\right]\right)$ be the intersection between the border of the polyomino and Rec (see Fig. 3). By considering the pixels of the border, the points A, A', B, B', C, C', D and D' delimit 8-connected curves of pixels which characterise the hv-convex polyomino. These 4 curves c1, c2, c3, and $\mathrm{c} 4$ are made of the points of the polyomino border being respectively located between $\mathrm{A}^{\prime}$ and $\mathrm{B}$ for $\mathrm{c} 1, \mathrm{~B}$ ' and $\mathrm{C}$ for $\mathrm{c} 2, \mathrm{C}^{\prime}$ and $\mathrm{D}$ for $\mathrm{c} 3$ and between $\mathrm{D}$ ' and A for $\mathrm{c} 4$, respectively.

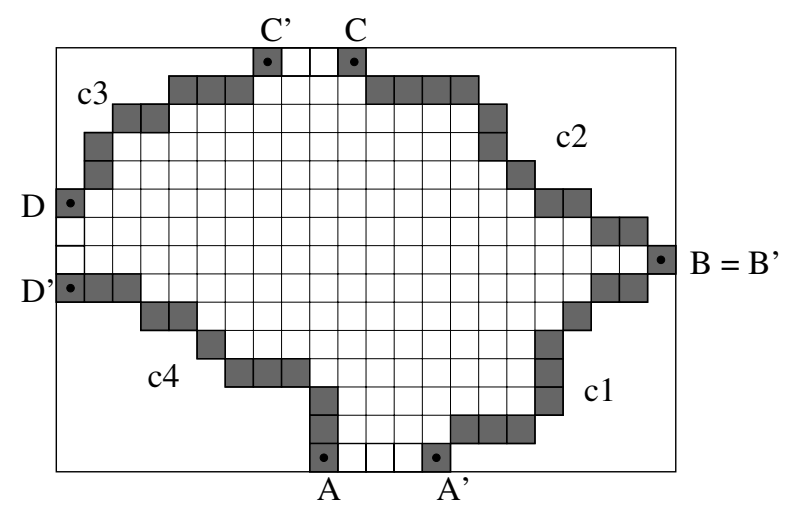

Fig. 3. A hv-convex polyomino, gray pixels represent the curves c1, c2, c3, and c4

As it has been indicated in section 2, to check the convexity of a hv-convex polyomino, we only have to consider the border; curves $\mathrm{c} 1, \mathrm{c} 2, \mathrm{c} 3$, and $\mathrm{c} 4$ must therefore be studied. The principle consists in segmenting curves $\mathrm{c} 1, \mathrm{c} 2, \mathrm{c} 3$, and $\mathrm{c} 4$, the eventual non-convexity of a polyomino shall be detected very simply through this operation. 
In the following paragraph, the notion of discrete line $[16,15,7]$ is recalled as well as some properties of these object types with, particularly, a new result on the construction of the convex hull of a discrete line segment. Moreover, an algorithm of discrete line segment recognition, with its use in the detection of convex polyominoes and its adaptation to the problem are presented.

\subsection{Discrete Lines}

The arithmetic definition of a discrete line was introduced by J.P. Reveillès $[16,15,7]$ :

Definition 1. A discrete line with a slope $\frac{a}{b}$ with $b \neq 0$ and $\operatorname{pgcd}(a, b)=1$, with lower bound $\mu$, arithmetical thickness $\omega$, is the set of points $(x, y)$ of $\mathbb{Z}^{2}$ which satisfies the double diophantian inequation

$$
\mu \leq a x-b y<\mu+\omega
$$

with all integer parameters.

We note the preceding discrete line $\mathcal{D}(a, b, \mu, \omega)$. We are mostly interested in naïve lines which verify $\omega=\sup (|a|,|b|)$ (see Fig. 4), we shall note them $\mathcal{D}(a, b, \mu)$. To simplify the writing, we shall suppose in the following that the slope coefficients verify $0 \leq a$ and $0 \leq b$, therefore $\omega=\max (a, b)$. Real straight lines $a x-b y=\mu$ et $a x-b y=\mu+\omega-1$ are named the leaning lines of the discrete naïve line $\mathcal{D}(a, b, \mu)$. An integer point of these lines is named a leaning point.

The leaning line located above (resp. under) $\mathcal{D}$ in the first quadrant $(0 \leq a$ and $0 \leq b)$ respects the following equation $a x-b y=\mu$ (resp. $a x-b y=\mu+\omega-1)$, it is named upper leaning line (resp. lower leaning line) of $\mathcal{D}$. All points of $\mathcal{D}$ are located between these two lines at a distance strictly lower than 1 . It is clear that every segment of a discrete line is a convex region.

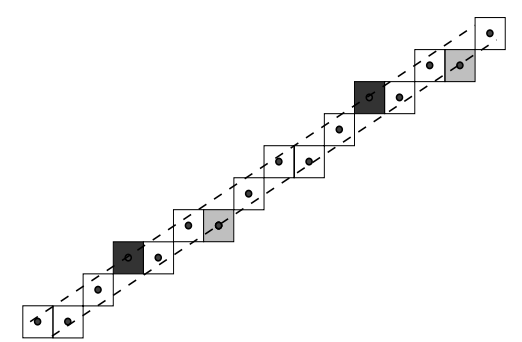

Fig. 4. A segment of the naïve line $\mathcal{D}(5,8,-1)$ for $x \in[0,15]$, the upper leaning points are in dark grey and the lower leaning points are in light grey, leaning lines are dotted lines

A naïve line $\mathcal{D}$ may be seen as a set of the integer points taken on the union of real lines $a x-b y=c$ with $c=\mu, \mu+1, \ldots, \mu+\omega-1$. We name $\alpha$-levelled 
dotted line of the line $\mathcal{D}$, the set of integer points located on the real line $a x-b y=\alpha$.

Definition 2. $r(M)$ is named the remainder at point $M\left(x_{M}, y_{M}\right)$ with respect to $\mathcal{D}$ and is defined by:

$$
\mathbf{r}(\mathbf{M})=\mathbf{a x}_{\mathbf{M}}-\mathbf{b y}_{\mathbf{M}} .
$$

Proposition 1. Let $M_{0} M_{1}$ be a segment of the line $\mathcal{D}(a, b, \mu)$, the lower convex hull of the $M_{0} M_{1}$ points is the polygonal curve going through the following points:

- The first and the last lower leaning point of the segment, named $L_{F}$ et $L_{L}$.

- Between $M_{0}$ and $L_{F}$ : the $\left(N_{i}\right)$ sequence with $x_{M_{0}} \leq x_{N_{i}} \leq x_{L_{F}}, N_{0}=M_{0}$ such that $x_{N_{i}}<x_{N_{i+1}}$ and $r\left(N_{i}\right)<r\left(N_{i+1}\right) \leq \mu+\omega-1$

- Between $M_{1}$ and $L_{L}$ : the $\left(P_{i}\right)$ sequence with $x_{M_{1}} \geq x_{P_{i}} \geq x_{L_{L}}, P_{0}=M_{1}$ such that $x_{P_{i}}>x_{P_{i+1}}$ and $r\left(P_{i}\right)<r\left(P_{i+1}\right) \leq \mu+\omega-1$.

(see Fig. 5 for an example).

\section{Remark 1.}

1. Colinear points of the $N_{i}$ and $P_{i}$ sequences may simply be omitted in the characterisation of the lower convex hull.

2. To construct the upper convex hull of a discrete line segment, the $N_{i}$ and $P_{i}$ sequences are determined in the same way from the upper leaning points.

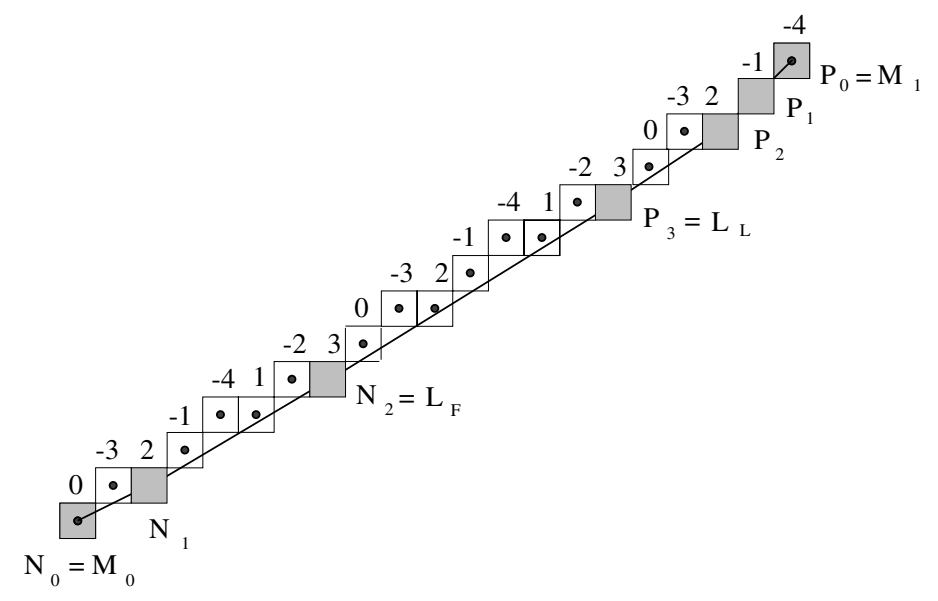

Fig. 5. Segment of the naïve line $\mathcal{D}(5,8,-4)$, the lower convex hull of the segment is drawn, the value of the remainder is indicated for each point 
Proof. All points of $\mathcal{D}$ are located above the lower leaning line, the segment points located between $L_{F}$ and $L_{L}$ are therefore above the segment $L_{F} L_{L}$ and the points $L_{F}$ and $L_{L}$ belongs to the lower convex hull of the segment.

Let us consider now the part of the segment between $M_{0}$ and $L_{F}$. Between these two points, we find at most $\omega-2$ points, with all different remainders. Let $N_{i}$ be a point of the sequence different of $L_{F}$, and $N_{i+1}$ the first point encountered since $N_{i}$ such that $N_{i+1}$ belongs to the interval $\left[r\left(N_{i}\right), \mu+\omega-1\right]$, the segment points between $N_{i}$ and $N_{i+1}$ are located above the dotted line going through $N_{i}$ and therefore above the segment $N_{i} N_{i+1}$.

Let $N_{i}, N_{i+1}, N_{i+2}$, be three consecutive points of the sequence. Let us prove that $N_{i+2}$ is located above or on the straight line $N_{i} N_{i+1}$, thus we shall have demonstrated the convexity of the sequence of points $N_{i}$. Two cases must be studied:

Case 1. If $r\left(N_{i+1}\right)-r\left(N_{i}\right) \leq r\left(L_{F}\right)-r\left(N_{i+1}\right)$ and $x_{L_{F}} \geq 2 x_{N_{i+1}}-x_{N_{i}}$ (see Fig. 6), the point $N_{i}^{\prime}$, symmetrical point of $N_{i}$ with respect to $N_{i+1}$, is a point on the segment located between $N_{i+1}$ and $L_{F}$. Let us suppose that $N_{i+2}$ is a point located between the dotted lines of level $r\left(N_{i+1}\right)$ and $r\left(N_{i}^{\prime}\right)$ such that $x_{N_{i+1}}<$ $x_{N_{i+2}}<x_{N_{i}^{\prime}}$. The symmetrical point $N_{i+2}^{\prime}$ of $N_{i+2}$ with respect to $N_{i+1}$ is a point of the segment whose remainder is included between $r\left(N_{i}\right)$ and $r\left(N_{i+1}\right)$ and whose x-coordinate is included between those of $N_{i}$ and $N_{i+1}$, which is contradictory with the hypotheses on $N_{i}$ and $N_{i+1}$. Necessarily $N_{i+2}=N_{i}^{\prime}$. Case $r\left(N_{i+1}\right)-r\left(N_{i}\right) \leq r\left(L_{F}\right)-r\left(N_{i+1}\right)$ and $x_{L_{F}}<2 x_{N_{i+1}}-x_{N_{i}}$ may not occur (property related to Klein Theorem and discrete lines [6]).

Case 2. If $r\left(N_{i+1}\right)-r\left(N_{i}\right)>r\left(L_{F}\right)-r\left(N_{i+1}\right), N_{i+2}$ is, by construction, a point located between the dotted lines of level $r\left(N_{i+1}\right)$ and $r\left(L_{F}\right)$ such that $x_{N_{i+1}}<$ $x_{N_{i+2}} \leq x_{L_{F}}$. Let us suppose that $N_{i+2}$ is located under the $N_{i} N_{i+1}$ line, by using the symmetrical point of $N_{i+2}$ with respect to $N_{i+1}$ we also get a contradiction. A similar reasoning may be applied to the sequence $\left(P_{i}\right)$ between $M_{1}$ and $L_{L}$.

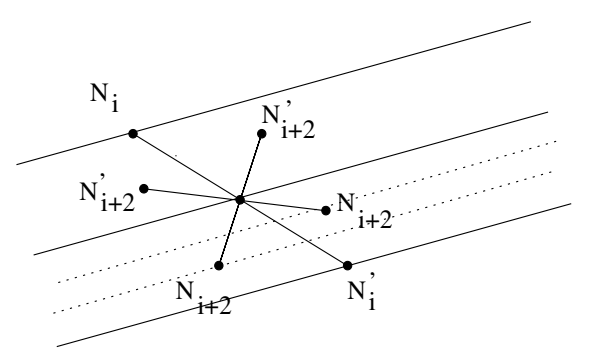

Fig. 6. Figure illustrating proof of Proposition 1 (Case 1) 


\subsection{Discrete Line Segment Recognition}

Let us consider $\Sigma\left(M_{0}, M_{1}\right)$ a segment of $\mathcal{D}$, discrete naïve line with characteristics $a, b, \mu, 0 \leq a$ and $0 \leq b, M_{0}$ and $M_{1}$ are respectively the first and the last point of the segment. Let us suppose that the point $M\left(x_{M}, y_{M}\right)$ (with $M=M_{1}+(1,0), M=M_{1}+(1,1)$ or $\left.M=M_{1}+(0,1)\right)$ is added to $\Sigma$. Is $\Sigma^{\prime}=\Sigma \cup\{M\}$ a straight line segment and, if it is the case, what are its characteristics $a^{\prime}, b^{\prime}, \mu^{\prime}$ ?

This problem is solved in $[7,6]$ and relies on some particular leaning points of a discrete line. They are the leaning points located at the extremities of the segment currently being recognised. We note $U_{F}$ (resp. $L_{F}$ ) the upper (resp. lower) leaning point whose $\mathrm{x}$-coordinate is minimal. In the same way, we note $\mathrm{U}_{\mathrm{L}}$ (resp. $\mathrm{L}_{\mathrm{L}}$ ) the upper (resp. lower) leaning point whose $\mathrm{x}$-coordinate is maximal.

Theorem 1 ( $[7,6])$. Let us consider $r(M)$, the remainder at point $M\left(x_{M}, y_{M}\right)$ with respect to $\mathcal{D}\left(r(M)=a x_{M}-b y_{M}\right)$.

(i) If $\mu \leq r(M)<\mu+\omega$, then $M \in \mathcal{D}(a, b, \mu), \Sigma \cup\{M\}$ is a segment of the straight line $\mathcal{D}$.

(ii) If $r(M)=\mu-1$, then $\Sigma \cup\{M\}$ is a segment of the straight line whose slope is given by the vector $\mathrm{U}_{\mathrm{F}} \mathrm{M}$.

(iii) If $r(M)=\mu+\omega$, then $\Sigma \cup\{M\}$ is a segment of the straight line whose slope is given by the vector $\mathrm{L}_{\mathrm{F}} \mathrm{M}$.

(iv) If $r(M)<\mu-1$ or $r(M)>\mu+\omega$, then $\Sigma \cup\{M\}$ is not a segment of a discrete line.

Remark 2. In the case $(i v) M$ is called strongly exterior to $\mathcal{D}$ and:

- If $r(M)<\mu-1$ ( $M$ is above $\Sigma$ ), there is an integer point between $\mathrm{U}_{\mathrm{F}} \mathrm{M}$ and $\Sigma$.

- If $r(M)>\mu+\omega(M$ is under $\Sigma)$, there is an integer point between $\mathrm{L}_{\mathrm{F}} \mathrm{M}$ and $\Sigma$.

The existence of these points is demonstrated at page 644 of [7].

Theorem 1 allows us to obtain an incremental algorithm (see Fig. 7) of discrete line segments recognition by scanning a sequence of 8-connected pixels named discrete path. A linear segmentation algorithm for 8-connected curves $[7,6]$ is immediately deduced from this result by considering the longest segments, last point of a segment being the first one of the next segment. To detect the convexity of a polyomino, we use a variant of this algorithm which is presented in the following section.

\subsection{Use of a Segmentation Algorithm for the Detection of Convex Polyominoes}

Detecting the convexity of a hv-convex polyomino P (see Fig. 3) consists in studying its convexity at the neighbourhood of each section of its borders $\mathrm{c} 1$, $\mathrm{c} 2, \mathrm{c} 3$, and $\mathrm{c} 4$. In this whole paragraph, convexity is described for the discrete curve $\mathrm{c} 1$, detection for the other curves is deduced by symmetry. 


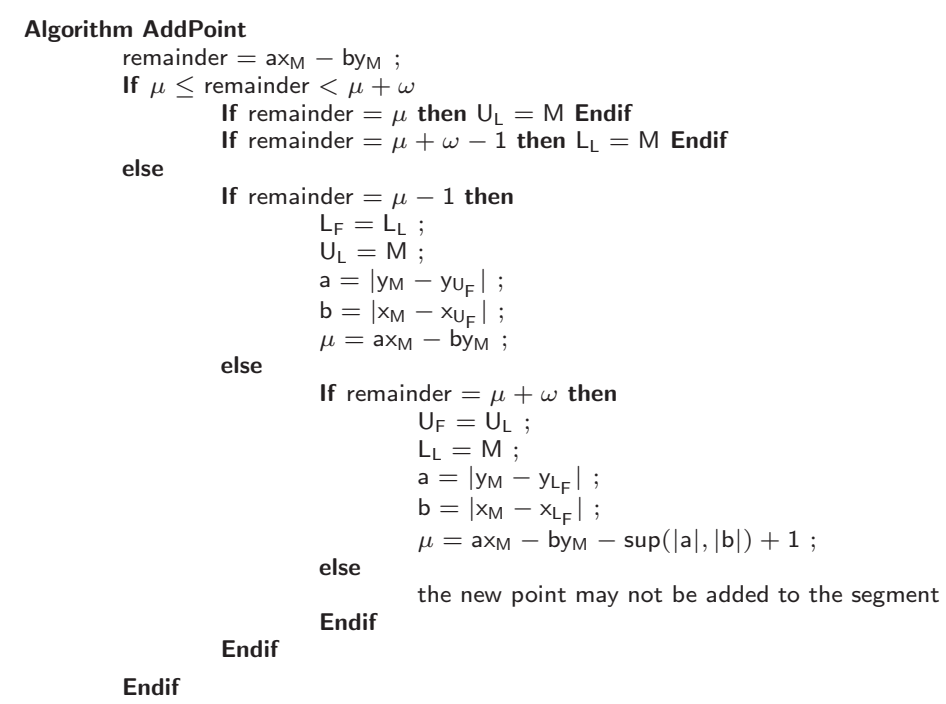

Fig. 7. Algorithm adding a point $M$ to the front extremity of a segment of a naïve line $\mathcal{D}(a, b, \mu)$ in the first quadrant

A curve is said lower convex if there is no discrete point between itself and its lower convex hull.

We must therefore prove that $\mathrm{c} 1$ is lower convex.

The algorithm for discrete line segments recognition is used on $\mathrm{c} 1$, it scans points of $\mathrm{c} 1$ one after another and stops when the added point may not belong to a discrete line segment containing all points already scanned plus the one added. The recognition shall not continue for a new segment at this added point but at the last lower leaning point of the segment which has just been recognised. When such a reject forces a segment change, the non convexity may be detected. The rejected point is located strongly above or strongly under the segment. Thanks to Remark 2, there is, in the second case, an integer point located between the discrete segment already scanned and the real segment from $M$ to the first lower leaning point. This segment to which $\mathrm{M}$ is added is therefore not lower convex and so neither is $\mathrm{c} 1$. In this case, the algorithm stops on a non-convexity statement of the polyomino.

Let us consider the procedure Recognizesegment $\left(M_{0}\right)$ whose input is a point $M_{0}$ of the curve $\mathrm{c} 1$ and which then adds the next points of $\mathrm{c} 1$ from $\mathrm{M}_{0}$ as long as the scanned set of points is a discrete line segment. This procedure outputs $M$, the last point tested, which is either the rejected point, or the last point of $\mathrm{c} 1$, the characteristics $(\mathrm{a}, \mathrm{b}, \mu)$ of the scanned segment with $a$ and $b$ positive, and the last lower leaning point $L_{L}$ as well as a boolean variable end, equal to false while c1 has still not been completely scanned. 
Definition 3. Let $S$ be a discrete line segment obtained by the procedure Recognizesegment, we call reduced segment of $S$ and we note $S$ ', the segment containing the points of $S$ located before the last lower leaning point existing in $S$, inclusive of this point.

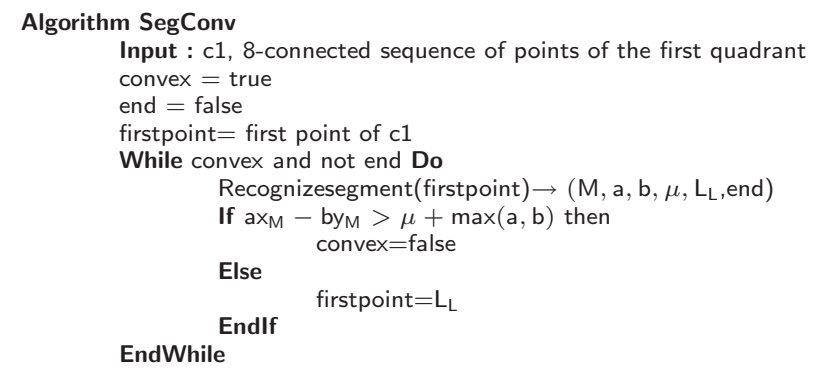

Fig. 8. Algorithm testing convexity of the part of a polyomino in the first quadrant

Theorem 2. A curve c1 of the first quadrant is lower convex if and only if the SegConv algorithm completely scans c1 (convex=true at the end).

Proof. Let us consider a point $M$ which may not be added to the current segment with characteristics $a, b, \mu$ such that $a x_{M}-b y_{M}>\mu+\max (a, b)$; then, according to Remark 2, there is an integer point between the current discrete segment and the straight line segment $L_{F} M$; therefore, according to the area property, c1 is not convex. In other words, when the algorithm stops because the variable convex has been set to false, $\mathrm{c} 1$ is not convex.

Let us suppose that $\mathrm{c} 1$ is completely scanned by the algorithm SegConv (see Fig. 8), then, all changes of straight line segments have been done on points $M$ which verify $a x_{M}-b y_{M}<\mu-1$. Each point is therefore located above the segment previously scanned.

Let S1 and S2 be two segments successively recognised by SegConv during the scanning of c1. S1 characteristics are $a_{1}, b_{1}, \mu_{1}$ and its slope is $\alpha_{1}\left(=\frac{a_{1}}{b_{1}}\right)$. Let us consider $L_{1 L}$ the last lower leaning point of S1 and $M$ the rejected point which does not belong to $\mathrm{S} 1$.

Let S'1 be the reduced segment of S1. Let us prove that the last edge of the convex hull of S'1 has a slope which is lower than the one of the first edge of the convex hull of $\mathrm{S} 2$.

As S'1 ends at the lower leaning point $L_{1 L}$ then, according to Proposition 1, if at least two lower leaning points are present in the segment, the slope of the last edge of the convex hull of S'1 is $\alpha_{1}$ otherwise, the slope is lower than $\alpha_{1}$.

Let $L_{1 L} K$ be the first edge of the convex hull of $\mathrm{S} 2$, whose slope is $\beta$, we must consider two cases:

- If $K \in \mathrm{S} 1$, necessarily $K$ is located above the lower leaning line of S1 therefore $\beta>\alpha_{1}$. 
- If $K \notin \mathrm{S} 1$, let us suppose that $\beta \leq \alpha_{1}$, i.e., that $K$ is under, or on, the lower leaning line of $\mathrm{S} 1$ (see Fig. 9). The points $L_{1 L}, M$, and $K$ belong to the segment S2. However that is impossible because the distance between $M$ and the leaning line of S1 is greater than 1, and so, a fortiori, the one between $M$ and $L_{1 L} K$. Therefore these three points may not belong to the same discrete line segment and $\beta>\alpha_{1}$.

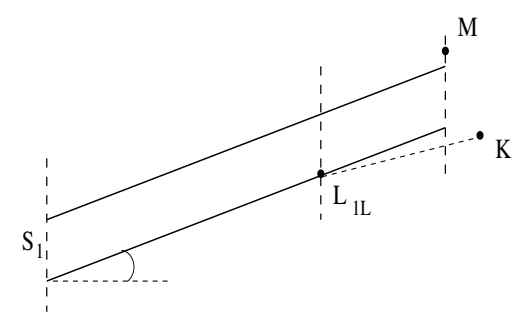

Fig. 9. Figure illustrating proof of Theorem 2

This property implies that the lower convex hull of the union of S'1 and S'2 is the union of the lower convex hulls of these both segments. As a generalisation, the lower convex hull of $\mathrm{c} 1$ is the union of convex hulls of the S'i. However each discrete segment is, by definition, lower convex. Therefore $\mathrm{c} 1$ is also lower convex, according to the definition of convexity by Kim and Rosenfeld (see Sect. 2) based on the convex hull of the set.

Complexity of the construction of curves $c_{i}, i=1 . .4$, is in $\mathrm{O}(m+n), m$ and $n$ being the numbers of rows and columns of the polyomino. Let us say a few words about the complexity of the SegConv algorithm. We first notice that the algorithm actually terminates. Indeed, at each step, the variable firstpoint is incremented by at least one element (in a non-void segment, $L_{L}$ is always different of the first element). On the other hand, the segmentation algorithm described in $[7,6]$ is linear in the number of points of the curve to scan. However, the variant we propose obliges to re-start every time at the last lower leaning point encountered, that leads to some overlaps. This algorithm is actually linear despite its overlaps. The proof of its linear complexity will be published in a next article.

\section{Applications to Discrete Tomography}

The objectives of discrete tomography is the reconstruction of a finite set of points from a number of projections. Several authors have been interested in this subject by using miscellaneous approaches to obtain exact, approached, or random solutions [9]. 
The particular case of polyominoes reconstruction from their horizontal and vertical projections is a NP-complete problem [19]. Moreover, if we suppose hconvexity (or v-convexity), the problems remains NP-complete [2]. On the other hand, if we consider hv-convex polyominoes, we get today several polynomial algorithms to decide if there is or not a polyomino corresponding to given projections $[2,3,5]$. The best existing algorithm is the one of Chrobak and Dürr [5]. This algorithm determines, for all couples of rows $k$ and $l$, if there is a hv-convex polyomino anchored in $k$ and $l$, with given horizontal and vertical projections. It is done by translating the existence of hv-convex polyominoes anchored in $k$ and $l$ as a 2SAT problem (conjunction of disjunctive clauses with at most two boolean variables), whose size is in $\mathrm{O}(m n)$. A 2 SAT problem may be solved linearly in its size [1] and the number of possible choices for $k$ and $l$ is $\min \left(m^{2}, n^{2}\right)$, as roles of rows and columns may be swapped. Therefore, it gives a complexity in $\mathrm{O}\left(m n \min \left(m^{2}, n^{2}\right)\right)$ for the reconstruction of a hv-convex polyomino with given vertical and horizontal projections. In fact, we may limit choices of $k$ and $l$, and then obtain an even more efficient implementation [5].

If we add to the algorithm of Chrobak and Dürr a convexity test, by using any method presented above, the complexity of the algorithm remains in $\mathrm{O}\left(m n \min \left(m^{2}, n^{2}\right)\right)$. On the other hand, if we want to decide if there is a convex polyomino with given projections by first seeking the hv-convex polyominoes having these projections, we shall have at worst an exponential complexity, because we can have an exponential number of hv-convex solutions [8].

We also have started to seek for the convex polyominoes with given orthogonal projections by using the paradigm of constraint programming. Let us give a short description of it:

1. A constraint program is the input

- of a priori domains in which variables of the problem are authorised to take their values,

- of constraints to which these variables are submitted,

- and of a strategy of assigning these variables.

2. The running of a constraint program consists in the repetition until a solution emerges, or as long as possibilities exist, of the following iteration:

- select, as a function of the prescribed strategy, a variable to assign, and an assignment value, among those in the domain of the variable which satisfy the constraints,

- update all constraints related to the variable which has just been assigned.

This iteration is associated, in case of a dead end (none of the variables remaining for assignment may be assigned), with a backtracking process, back to the last choice point.

This approach had already been used for the detection of the existence of hvconvex polyominoes with given orthogonal projections [20]. We have [17] modified the program written at this occasion, by adequately enriching the set of constraints. 
This new program has successfully run on a first sample of data. Moreover, we have observed the following: if we just take care of how to express the constraints, the adopted process allows us to take them into account globally, rather than successively. So, we avoid to apply first constraints related to the hv-convexity, and then the ones related to the convexity itself. This global treatment allows us to obtain convex polyominoes more quickly, when they exist, without having to enumerate previously the hv-convex polyominoes, which may be more numerous. And in the same way, it is possible to decide more rapidly that none of them exists, when it is the case.

These first results are encouraging and push us to deepen this approach.

\section{Conclusion}

Our work has permitted a step forward in two directions.

On the one hand, we obtain a characterisation of lower and upper convex hulls of a discrete line segment based on the arithmetic interpretation of these objects.

On the other hand, we obtain a linear algorithm to test the convexity of a polyomino, which is very simple to express. We prove the correctness of this algorithm. After this paper was prepared, the authors' attention was called by a reviewer to Hübler, Klette and Voß' paper [10]. We will compare their approach and ours in our oral presentation.

Moreover, we have explored an alternative approach of the convexity test, in particular by using the methods of constraint programming. We have also applied the convexity test to some problems of discrete tomography. In both cases, the first results obtained urge us to continue on this way.

\section{Acknowledgements}

We thank the members of the PolKA research group of LORIA for their review and constructive remarks.

\section{References}

1. B. Aspvall, M. F. Plass, and R. E. Tarjan. A linear-time algorithm for testing the truth of certain quantified boolean formulas. Information Processing Letters, volume 8, number 3, pages 121-123, 1979. 502

2. E. Barcucci, A. Del Longo, M. Nivat, and R. Pinzani. Reconstructing convex polyominoes from horizontal and vertical projections. Theoretical Computer Science, volume 155, number 2, pages 321-347, 1996. 502

3. E. Barcucci, A. Del Longo, M. Nivat, and R. Pinzani. Medians of polyominoes: A property for the reconstruction. International Journal of Imaging Systems and Technology, volume 8, pages 69-77, 1998. 502

4. J-M. Chassery, A. Montanvert. Géométrie discrète en analyse d'images. Traité des nouvelles technologies, série Images, Hermes, 1991. 491, 493 
5. M. Chrobak and C. DürR. Reconstructing hv-convex polyominoes from orthogonal projections. Information Processing Letters, volume 69, pages 283-289, 1999. 502

6. I. Debled-Rennesson. Etude et reconnaissance des droites et plans discrets. Thèse. Université Louis Pasteur, Strasbourg, 1995. 491, 497, 498, 501

7. I. Debled-Rennesson and J. P. Reveillès. A linear algorithm for segmentation of digital curves. In International Journal of Pattern Recognition and Artificial Intelligence, volume 9, pages 635-662, Décembre 1995. 491, 495, 498, 501

8. A. Del Lungo and M. Nivat and R. Pinzani. The number of convex polyominoes reconstructible from their orthogonal projections. Discrete Mathematics, volume 157, pages $65-78,1996.502$

9. G. T. Herman and A. KubA. Discrete Tomography. Birkhauser, 1999. 501

10. A. Hübler, R. Klette and K. Voß. Determination of the Convex Hull of a Finite Set of Planar Points Within Linear Time. Elektronische Informationsverarbeitung und Kybernetik, pages 121-139, 1981. 503

11. C. E. KIM. Digital convexity, straightness and convex polygons. In IEEE Transactions on Pattern Analysis and Machine Intelligence, volume PAMI-4, pages 618626, 1982. 492

12. C. E. Kim and A. Rosenfeld. On the convexity of digital regions. In Pattern Recognition, volume 5, pages 1010-1015, 1980. 492

13. C. E. Kim and A. Rosenfeld. Digital straight lines and convexity of digital regions. In IEEE Transactions on Pattern Analysis and Machine Intelligence, volume PAMI-4, pages 149-153, 1982. 492

14. M. Minsky and S. Papert. Perceptrons. In M. I. T. Press, 1969. 492

15. J. P. Reveillès. Géométrie discrète, calculs en nombre entiers et algorithmique. Thèse d'état. Université Louis Pasteur, Strasbourg, 1991. 495

16. J. P. REveillès. Structure des droites discrètes. In Journées mathématique et informatique, Marseille-Luminy, Octobre 1989. 495

17. I. Sivignon. Reconstruction de polyominos convexes par programmation par contraintes. Rapport du stage de Magistère d'Informatique 1ère année, ENS Lyon, effectué au LORIA, 1999. 502

18. J. Slansky. Recognition of convex blobs. In Pattern Recognition, volume 2, pages 3-10, 1970. 492

19. G. J. Woeginger. The reconstruction of polyominoes from their orthogonal projections. Technical report, Technische Universität Graz, 1996. 502

20. T. Zajac. Reconstructing Convex Polyominoes using Constraint Programming. Master Thesis, Wroclaw University of Technology, Pologne. 502 